\title{
Evaluation of Scatter Suppression Algorithm for X-ray Exposure of Soft Tissue Equivalent Phantoms Over Nominal Energy Range Using FLUKA Code
}

\author{
Samson O. Omondi ${ }^{1,2 *}$, Innocent J. Lugendo ${ }^{2}$, Ramadhan R. Kazema ${ }^{3}$ and Robert Kinyua ${ }^{4}$ \\ ${ }^{l}$ Department of Radiography, College of Health Sciences, Jomo Kenyatta University of \\ Agriculture and Technology, Nairobi, Kenya \\ ${ }^{2}$ Department of Physics, University of Dar es Salaam, Tanzania \\ ${ }^{3}$ Department of Radiology and Imaging, Muhimbili University of Health and Allied Sciences, Dar \\ es Salaam, Tanzania \\ ${ }^{4}$ Department of Physics, Jomo Kenyatta University of Agriculture and Technology, Nairobi, Kenya \\ *Corresponding author, E-mail: samomondi@jkuat.ac.ke; samkotieno@yahoo.com \\ Co-authors'e-mail addresses: ilugendo26@gmail.com; rkazema@hotmail.com; \\ robertkinyua@jkuat.ac.ke
}

Received 12 Feb 2021, Revised 24 May 2021, Accepted 27 May 2021, Published May 2021

DOI: https://dx.doi.org/10.4314/tjs.v47i2.33

\begin{abstract}
Soft tissue imaging is heavily impaired by streaks and cupping effects associated with X-ray scatter. Quality of images from projection imaging may be improved by the use of enhanced antiscatter grids' designs with potency to reject significant scatter. However, optimization of grid characteristics requires investigation to improve diagnostic image quality. Transmitted scatter spatially distributed degrades images engendering need for effective scatter correction protocols. This study investigated the pre-scan scatter suppression algorithm for X-ray exposure of soft tissue equivalent phantoms over nominal energy range. Adipose tissue and polymethyl methacrylate phantoms of cross-sectional area $(30 \times 30) \mathrm{cm}^{2}$ and of varying thickness from 2 to $8 \mathrm{~cm}$ in $1 \mathrm{~cm}$ increments were successively exposed using energy ranging between $20-50 \mathrm{kVp}$. Monte Carlo simulation based on FLUKA code and flair interface was used to generate an input file for execution. The source simulated five cycles of ten million photons each of annular X-ray photon beam of radius, $\mathrm{r}=0.5 \mathrm{~cm}$ at fixed field of view (FOV) through anti-scatter grid on to gadolinium oxysulfide detector. The transmitted total, scatter and primary estimates were evaluated with and without grids over varying phantom thicknesses, energy and grid design features. The simulated and experimental results obtained were comparable and in agreement with previous literature. Pearson's correlation coefficients for scatter fraction and scatter to primary ratio were 0.983 and 0.981 , respectively. The strong correlation between simulation and experiment results indicated correctness in methodology and protocol. The algorithms and protocols in the simulation would be appropriate for designing grids with enhanced scatter rejection capabilities.
\end{abstract}

Keywords: FLUKA code, Monte Carlo simulation, Scatter suppression algorithm, Scatter correction, X-ray imaging systems.

\section{Introduction}

The use of X-ray based digital imaging systems has become indispensable for medical diagnosis notwithstanding non-ionizing radiation modalities entry in radiology departments. However, the attenuation maps/images produced by $\mathrm{X}$-ray radiations are often corrupted by inherent scattering during 
interactions with human tissues. The evaluation of possible Compton and Raleigh scattering effects from diagnostic radiation will only be possible once the scatter estimation and correction methods and algorithms are well investigated (Sisniega et al. 2013, Stankovic et al. 2017). Radiation interactions in matter undergo random and stochastic mechanisms resulting in scattering. Compton and multiscattering dominate interactions in biological tissues/equivalent phantoms with a small contribution from Raleigh scattering (Wang et al. 2012, Del Lama et al. 2017). These interactions result in degradation to the diagnostic image quality. Inferior image quality impairs visualization of intended pathology thereby reducing validity of clinical decisions (Omondi et al. 2020).

Several degrading mechanisms exist, including patient voluntary and involuntary motion, detector blur, inferior detective quantum efficiency (DQE) and scatter radiations amongst others. Scatter radiation being the dominant image degradation mechanism, many researchers have investigated hardware based scatter correction methods that currently refer to the use of antiscatter grids (Fetterly and Schueler 2008, Carton et al. 2009, Zhou et al. 2016, Omondi et al. 2020). However, the scatter correction efficacy of anti-scatter grids needs further improvements by improving the grid designs based on the fundamental grids features that include, grid ratio $(r)$, grid frequency $(N)$ and septa to interspace ratio $(R)$ amongst others (Zhou et al. 2020). Experimental and analytical results often suffer several inconsistencies and inaccuracies. Their acceptance requires deliberate efforts to compare them over more accurate, robust and computational efficient methods (Chan et al. 1990, Zhou et al. 2018). Thus, the experimental results of polymethyl methacrylate (PMMA) exposure would be used to validate the FLUKA code simulation results. The aim of this study was to evaluate scatter suppression algorithm for X-ray exposure of soft tissue equivalent phantom PMMA over nominal mammographic energy ranges of 20 -
$49 \mathrm{kVp}$ by Monte Carlo simulation based on FLUKA code.

\section{Materials and Methods \\ Anti-scatter grids}

In this study, commercial and simulation generated parallel focused anti-scatter grids of varying grid designs (mainly grid ratio and septa frequency) were investigated for optimal scatter rejection. The following equations as given by Chan and Doi (1982) were used to determine the grid characteristics for input in the imaging geometry for this study. A user defined $\mathrm{C}++$ code was written that enabled the input of the variables that generated the grid designs into the input file's combinatorial geometry (CG). The expressions are as given in equations $1-4$;

$$
\text { Grid ratio, } r=h / D
$$

Grid frequency (lines/cm), $N=1 / d+D$

Septa to inter-space ratio, $R=d / D$

Lead content $\left(\mathrm{gcm}^{-2}\right), P=\rho N h d$

where, $d$ is the septa thickness, $D$ is the interspace distance, $h$ is the grid height and $\rho$ is the density of the septa material $=11.34 \mathrm{~g} \mathrm{~cm}^{-}$ 3. The anti-scatter grid's position in the imaging geometry is critical in limiting the deviation and further interactions of the transmitted primary estimate.

\section{Monte Carlo simulation: The FLUKA particle transport code}

The FLUKA particle transport code is an open source coupling advanced graphical user interface flair was found appropriate for this work. This code is computationally efficient in generating particle transport and interactions histories, can track a wide range of charged and neutral particles including photons and electrons with energies ranging from $100 \mathrm{eV}$ to $\sim 1000 \mathrm{TeV}$ (Ferrari et al. 2005). This code is suitable for diagnostic imaging applications because it uses original transport algorithm for charged particles including complete Multiple Coulomb scattering treatment giving the 
correct lateral displacement even near the boundary. FLUKA can handle and support complex geometries using an inbuilt CG package and provides options for user written routines for customizing simulations for scatter radiation estimation (Vlachoudis 2009). The source is placed along the iso-centre perfectly aligned in the geometry as shown in Figure 1.
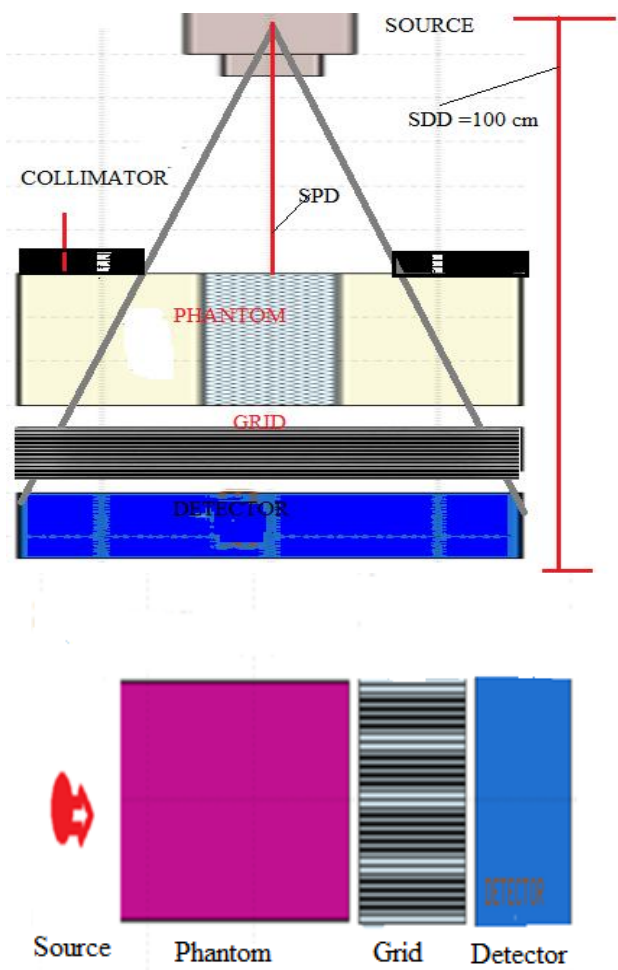

Figure 1: Experimental and simulation imaging geometry.

The simulation protocol

The FLUKA code has an inbuilt combinatorial geometry (CG) that allowed the construction of the simulation geometry as shown in Figure 1. The choice of CG depended on the complexity and body section and/or tissue to be reconstructed as a phantom for irradiation. The anti-scatter grid designs were generated using the Equations 1-4 on a user written code (subroutine). The code allowed successive inputs of the septa thickness, interspace distance, septa material density, and grid height. These inputs enabled the constructions of varying antiscatter grids positioned between the phantom and the detector. The source position was maintained for general and mammographic applications as in the Table 1 . The transmitted $\mathrm{X}$-radiations were detected on a sensitive detector; gadolinium oxysulfide $\left(\mathrm{GD}_{2} \mathrm{O}_{2} \mathrm{~S}\right)$ as fluence flux, energy deposits and photon dose per region of the geometry. The simulation of the X-ray transport process was done using nominal photon energy ranges $20-49 \mathrm{kVp}$ for PMMA and adipose tissue (mammographic range). The number of photons was set at 10 million to be run over 5 run cycles to generate photon histories and simulation projection data for analysis. Two sets of data were obtained. The first set without the primary beam stopper in place to register the total photon transmissions $(I t)$. The second set was acquired with the primary beam stopper in place registering in the detector the spatially distributed scatter transmitted estimate $(I s)$. The energy/fluence deposited on the detector was used to compute scatter distribution. And lastly, extraction of the image/primary estimate (Ip) was done by subtracting the scatter estimate $(I s)$ from the raw projection data $(I t)$ in the spatial domain $(x, y)$ using the expression:

$$
I_{p}(x, y)=I_{t}(x, y)-I_{s}(x, y)
$$

\section{MCs projection data acquisition}

The MCs method acquires scatter distribution from the results of particle transport by utilizing a binning mechanism that allows for collection of millions of data. The data in binary files were compiled and processed into numerical data for further analysis. USRBIN and USRTRACK cards were used to acquire MCs data in executable or binary files. The data were compiled and processed generating GNU plots for the energy deposited, photon dose and fluence on the detector. An estimate of scatter intensity was determined by simulating the physical path of transmitted and 
Omondi et al. - Evaluation of scatter suppression algorithm for X-ray exposure of soft tissue ...

scattered photons through the object, antiscatter grids into the image detector. A total of 10 million photons were impinging through the iso-center of the phantom in 5 cycles for each run. The generated files and data were analyzed using integrated software.

Table 1: Simulation parameters and geometry values

\begin{tabular}{lll}
\hline Parameter & Mammographic values & Digital radiography values \\
\hline Source point & $0,0,-60$ & $0,0,-60$ \\
Primary particles number & $10^{7}$ & $10^{7}$ \\
X-ray spectra energy & $20-50 \mathrm{kVp}$ & $50-150 \mathrm{kVp}$ \\
Source axis distance (SAD) & $30 \mathrm{~cm}$ & $65 \mathrm{~cm}$ \\
Source detector distance (SDD) & $65 \mathrm{~cm}$ & $120 \mathrm{~cm}$ \\
Detector matrix & $3020 \times 3800$ & $1024 \times 1024$ \\
Detector pixel size & $0.005 \mathrm{~cm}$ & $0.05 \mathrm{~cm}$ \\
Phantom material & PMMA & Bone equivalent phantom \\
Detector material & Gadolinium $\left(\mathrm{GD}_{2} \mathrm{O}_{2} \mathrm{~S}\right)$ & Gadolinium $\left(\mathrm{GD}_{2} \mathrm{O}_{2} \mathrm{~S}\right)$ \\
USRBIN card & Scores fluence, energy & Fluence and energy \\
USRTRACK card & Particle tracks \& fluence & Fluence tracks \& fluence \\
\hline
\end{tabular}

\section{Scatter estimation algorithm}

Analysis of photons arriving at the detector was based on several assumptions, some of which are; uniform linear attenuation characteristics of the annular region of phantom/object, a constant photon energy during the simulations and experiments, uniform spatial distribution of photons in the pixels, characteristics of the anti-scatter grids amongst others. Consider Xray radiations after interactions with the phantom/object arriving at the detector in the spatial distribution $(x, y)$; primary signals $(P s)$ and scatter signals $(S s)$, respectively. The incident intensity at the detector with and without anti-scatter grids was determined using the Equations 6 and 7;

$I_{W G}(x, y)=\Upsilon\left(P_{s}(x, y)+s_{s}(x, y)\right)$

$I_{G}(x, y)=\Upsilon\left(I_{p} P_{s}(x, y)+I_{s} s_{s}(x, y)\right)$

where, $\Upsilon$ is the proportionality constant relating to the incident photon intensity to the detector signal, $I_{W G}$ and $I_{G}$ are intensities at the detector without grid and with grid in geometry, respectively. Is represents scatter signals, and Ip represents the transmitted primary/image signals.

\section{Scatter measurements}

PMMA breast tissue equivalent phantom of varying thickness (2 to $8 \mathrm{~cm}$ ) of cross-sectional area $30 \times 30 \mathrm{~cm}^{2}$ was exposed with and without the anti-scatter grids. All measurements were acquired using a $20-49 \mathrm{kVp}$ and $50-110 \mathrm{kVp}$ $\mathrm{X}$-ray beam for bone tissue equivalent phantom (B-00). The transmitted total photons (It) and scatter $(I s)$ were used to calculate primary (Ip) photons according to Equation 5. These measurements were repeated for different antiscatter features, especially grid ratio and septa frequency. MCs of scattered signals to estimate the scatter fraction in detected intensities was recorded as photon fluence, dose or deposited energy on the detector representing the photons transport through the object. The scattered photons are low spatial frequency signals spatially distributed over the detector surface. The simulated and experimental scatter estimates were compared. From the results of the scatter measurements, two photons transmission related parameters were determined, namely scatter fraction (SF) and scatter to primary ratio (SPR). The scatter measurement took into account, the grid characteristics, number of scattered photons, the photon histories and the deposited energy in the detector. 


\section{Signal to noise ratio (SNR)}

In digital X-ray diagnostic imaging modalities, incident exposure when anti-scatter grid is used needs not to be increased because the primary/image signals may be amplified by optical or electronic adjustments of the flat panel detectors (FPDs) system gain before being processed and displayed. For digital imaging systems, use of anti-scatter grid should not cause an increase in patient dose. However, effects of anti-scatter grids on the SNR due to quantum noise in digital radiography have been discussed in literature. SNR was determined using the expression;

$$
S N R=\frac{S}{\sqrt{N_{q}^{2}+N_{S}^{2}}}
$$

where $N_{S}$ is detector system noise (dependent on electronic noise and digitization noise), $N_{q}$ is the quantum noise and $S$ is the signal of interest in the image (Salvagini et al. 2012).

\section{Ethical consideration}

Muhimbili National Hospital Research Ethics Committee (MNH-REC Reference: MNH/TRCU/permission/2020/123 and ethical clearance certificate number MNH/IRB/1/2020/007) were granted to the authors to access the Radiology Departments of both Mloganzila and Upanga MNH facilities.

\section{Results and Discussion \\ Results}

Anti-scatter grids performance in scatter corrections

Generated anti-scatter grid designs based on Equations 1-4 are presented in Table 2. Three anti-scatter grids N31r5, N36r5 and N40r8 shown in Table 2 were found to have been used in previous studies. From Table 2, 11 grids with grid ratios ranging between 5 and 21 were investigated for scatter photons transmission suppression efficacy in digital radiography units.

Table 2: Generated grid designs from a user defined code from combinatorial geometry (CG) of flair of FLUKA MCs code.

\begin{tabular}{llllllll}
\hline Grid reference & $\mathrm{r}$ & $\mathrm{h}(\mathrm{mm})$ & $\mathrm{D}(\mu \mathrm{m})$ & $\mathrm{d}(\mu \mathrm{m})$ & $\mathrm{R}$ & $\mathrm{P}\left(\mathrm{g} / \mathrm{cm}^{2}\right)$ & $\mathrm{N}\left(\right.$ lines cm $\left.^{-1}\right)$ \\
\hline N31r5 & $5: 1$ & 1.50 & 300 & 2.0 & 0.0067 & 0.1126 & $31^{\#}$ \\
N36r5 & $5: 1$ & 1.3 & 257 & 2.0 & 0.0078 & 0.1125 & $36^{*}$ \\
N38r6 & $6: 1$ & 1.7 & 283 & 3.0 & 0.0106 & 0.2022 & 38 \\
N34r8 & $8: 1$ & 2.0 & 250 & 2.1 & 0.0084 & 0.1890 & 34 \\
N40r8 & $8: 1$ & 1.7 & 288 & 2.3 & 0.0080 & 0.2066 & $40^{+}$ \\
N44r10 & $10: 1$ & 2.5 & 250 & 4.5 & 0.0180 & 0.5013 & 44 \\
N44r11 & $11: 1$ & 3.0 & 273 & 8.0 & 0.0293 & 0.9685 & 44 \\
N25r12 & $12: 1$ & 2.8 & 233 & 3.0 & 0.0129 & 0.4036 & 25 \\
N30r15 & $15: 1$ & 3.5 & 233 & 1.2 & 0.0052 & 0.2034 & 30 \\
N40r5 & $16: 1$ & 5.0 & 313 & 3.4 & 0.0109 & 0.6093 & 40 \\
N36r21 & $21: 1$ & 5.0 & 238 & 4.5 & 0.0189 & 1.0522 & 36 \\
\hline
\end{tabular}

*Omondi et al. 2020, " Carton et al. 2009 and ${ }^{+}$Fetterly and Schueler 2007. Anti-scatter grid designs used in previous studies. The values were obtained using Equations 1-4 generated from the user code.cpp run on flair input file.

From Table 3, SPR and SF values both from simulated and experimental designs are presented. The SPR and SF values slightly increased with the phantom thickness which results from greater interactions generating much scatter as the phantom thickness increases. Table 3, presents the grid performance parameters SF and SPR simulated and experimental results across varying phanton thickness $\mathrm{t}, 2 \mathrm{~cm} \leq \mathrm{t} \leq 8 \mathrm{~cm}$.

Figures $2(a, b)$ show the error bar plots of simulated and experimental values for SPR, and SF showed near unity correlations with Pearson's r, values of 0.981 and 0.983 , 
Omondi et al. - Evaluation of scatter suppression algorithm for X-ray exposure of soft tissue ...

respectively. This indicates that the results from the two methods are within confidence interval of 0.5 and validated by the experimental results and methodology. For Raleigh and Compton scattering conditions, the combinations of energy in $\mathrm{kV}$, septa frequency and grid ratio have been found for which a well-designed and aligned anti-scatter grid result in the lowest mean scatter photons transmission. Low scatter transmission yields improve the SNR improvement factor.

Table 3: Experimental and simulation SF and SPR values at varying PMMA thickness at fixed nominal energy $(34 \mathrm{kVp})$.

\begin{tabular}{|c|c|c|c|c|c|c|c|c|}
\hline \multicolumn{2}{|c|}{ PMMA thickness $(\mathrm{cm})$} & 2 & 3 & 4 & 5 & 6 & 7 & 8 \\
\hline \multirow[t]{2}{*}{ SF } & Experimental \pm 0.002 & 0.255 & 0.263 & 0.272 & 0.285 & 0.303 & 0.314 & 0.332 \\
\hline & Simulation $\quad \pm 0.001$ & 0.257 & 0.261 & 0.271 & 0.283 & 0.301 & 0.315 & 0.334 \\
\hline \multirow[t]{2}{*}{ SPR } & Experimental \pm 0.002 & 0.337 & 0.343 & 0.372 & 0.384 & 0.420 & 0.443 & 0.489 \\
\hline & Simulation & 0.335 & 0.341 & 0.367 & 0.382 & 0.418 & 0.445 & 0.484 \\
\hline
\end{tabular}
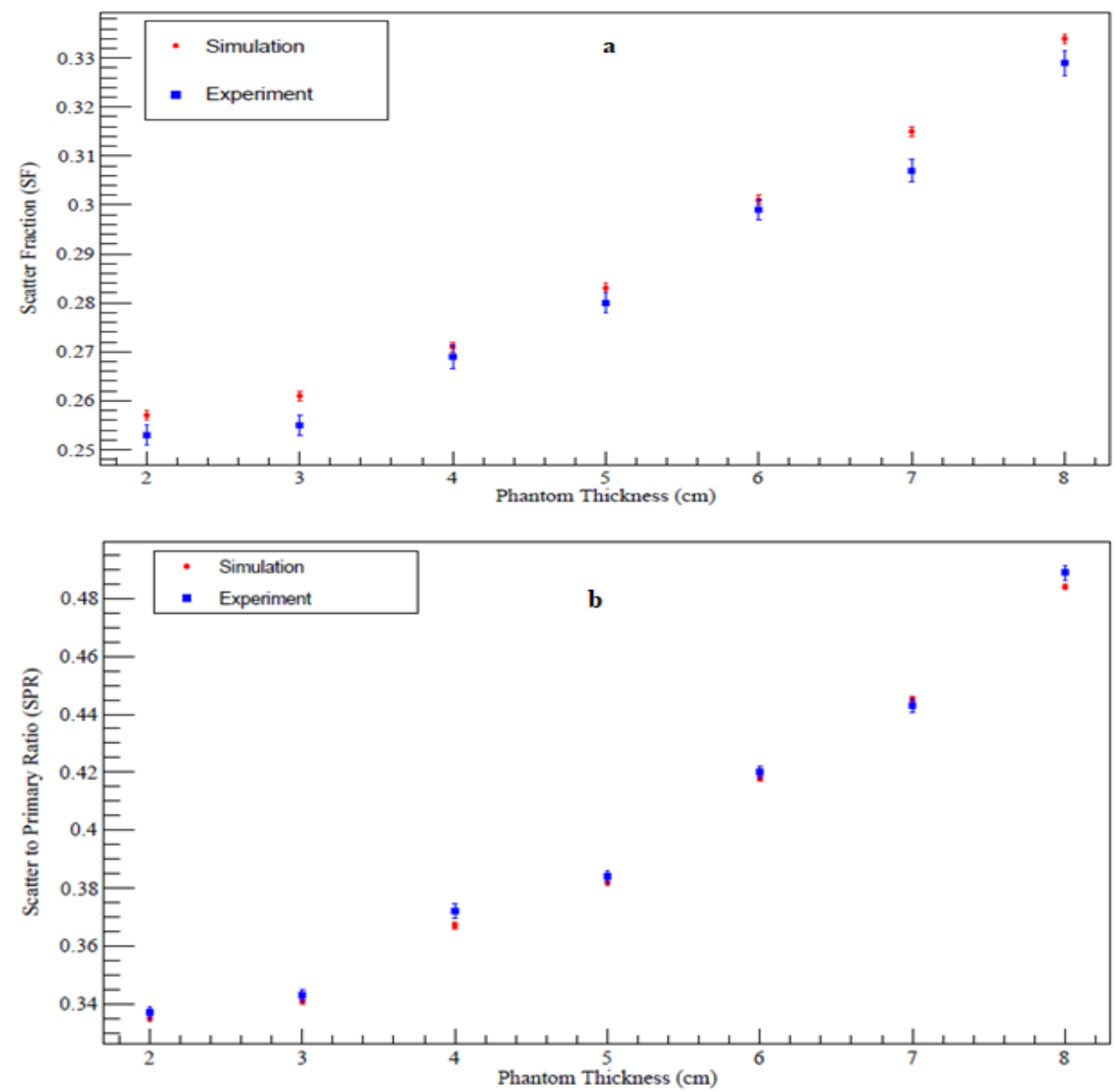

Figure 2 (a, b): Simulated and experimental values of SF and SPR against PMMA phantom thickness at fixed X-ray energy $(34 \mathrm{kVp})$. 
Figure 3 shows the fluence intensity of transmitted radiations through the regions of the phantom, anti-scatter grids and the detector for narrow beam conditions. Narrow beams result in lower spatial distribution of scatter on the detector. Narrow beams transmit primary photons carrying high intensity fluence through the object/phantom. The sparsely distributed energy deposits over the detector region are transmitted scatter about the central axis. The primary plus scatter estimates give the total energy deposits on the $\mathrm{GD}_{2} \mathrm{O}_{2} \mathrm{~S}$ detector. The bone equivalent plastic phantom (B-100) absorbs low energy scatter photons that remain within the object/phantom (residual doses) and almost uniformly spatially distributed over the phantom region.

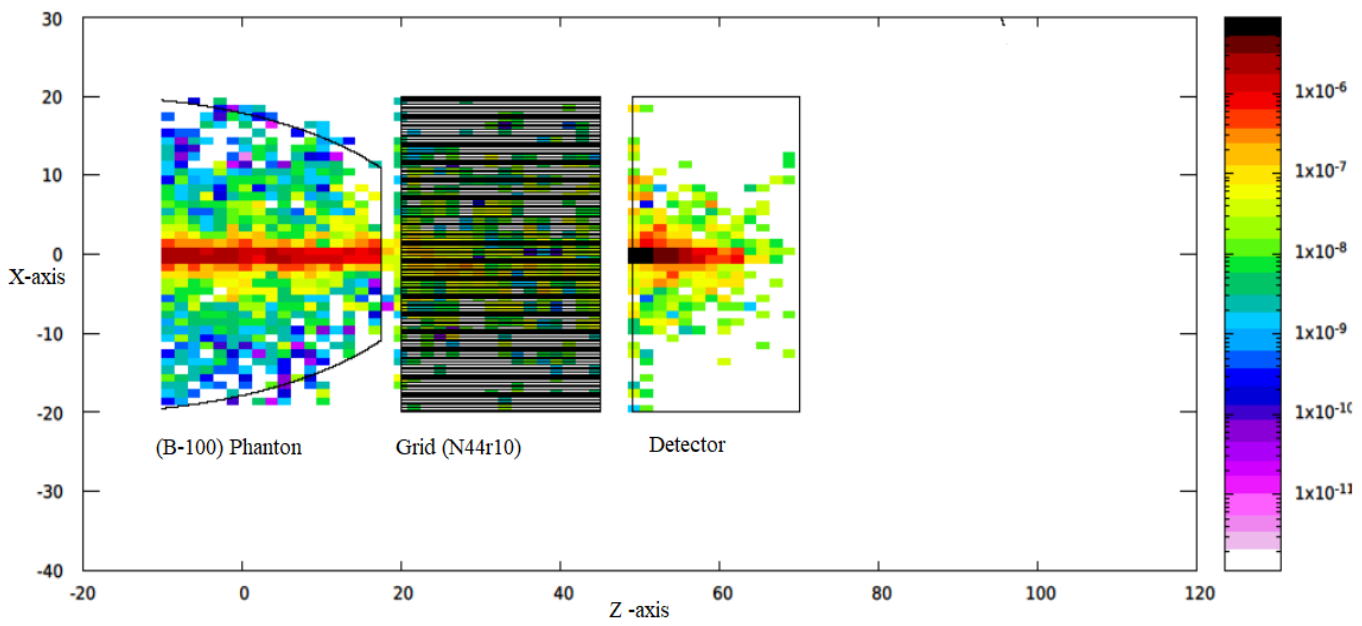

Figure 3: $2 \mathrm{D}$ GNU plots of fluence intensity spatial distribution per region of geometry for a bone equivalent phantom (B-100) irradiation through the anti-scatter grid to the detector.

From Figure 3, the fluence intensities per region of the simulation geometry are presented. The tissue attenuates and absorbs low energy photons, the anti-scatter grids region records mixed X-ray photons energy, while the image forming transmitted primary photons of higher energy peaks are registered at the detector.

From Table 4, results from three previous studies have been presented alongside the results from this study. This study however, investigated the thickness of phantom $t$, in 1 $\mathrm{cm}$ increments from 2 to $8 \mathrm{~cm}$. Statistical analysis of the data in Table 4 yielded $\mathrm{R}^{2}$ value of 0.997 and Pearson's correlation coefficient $\mathrm{r}$, of 0.9987 , indicating that the results are comparable and in agreement with literature thus validating our methodology.

Table 4: Comparing SPR results of this study and literature.

\begin{tabular}{lllllllll}
\hline Previous studies & \multirow{2}{*}{ Energy $(\mathrm{kVp})$} & \multicolumn{7}{c}{ Thickness of PMMA phantom (cm) } \\
\cline { 3 - 8 } & & 2 & 3 & 4 & 5 & 6 & 7 & 8 \\
\hline Salvagnini et al. (2012) & $34 \mathrm{kV}$ & 0.32 & - & 0.61 & - & 0.88 & - & 1.24 \\
Zhou et al. (2016) & $34 \mathrm{kV}$ & 0.31 & - & 0.60 & - & 0.88 & - & 1.15 \\
Del Lama et al. (2017) & $34 \mathrm{kV}$ & 0.28 & - & 0.56 & - & 0.82 & - & 1.08 \\
This study & $34 \mathrm{kV}$ & 0.29 & 0.47 & 0.59 & 0.78 & 0.85 & 0.94 & 1.19 \\
\hline
\end{tabular}


Figures 4 and 5 show the profiles across the simulated geometry. The unfolded simulated spectrum in Figure 4 represents the raw attenuations through varying phantom thickness at photon peak enegies. Figure 5 presents convolved peaks of deposited energies across the simulation geometry, the phantom, anti-scatter grid and the detector. The scatter radiation estimates cut-off at the grid amplifies the amplitude of the photons energy deposits. The primary/image peaks are shown in the high amplitude peaks towards higher photon energies. The image carrying, scatter corrected primary estimate is of higher intensity to

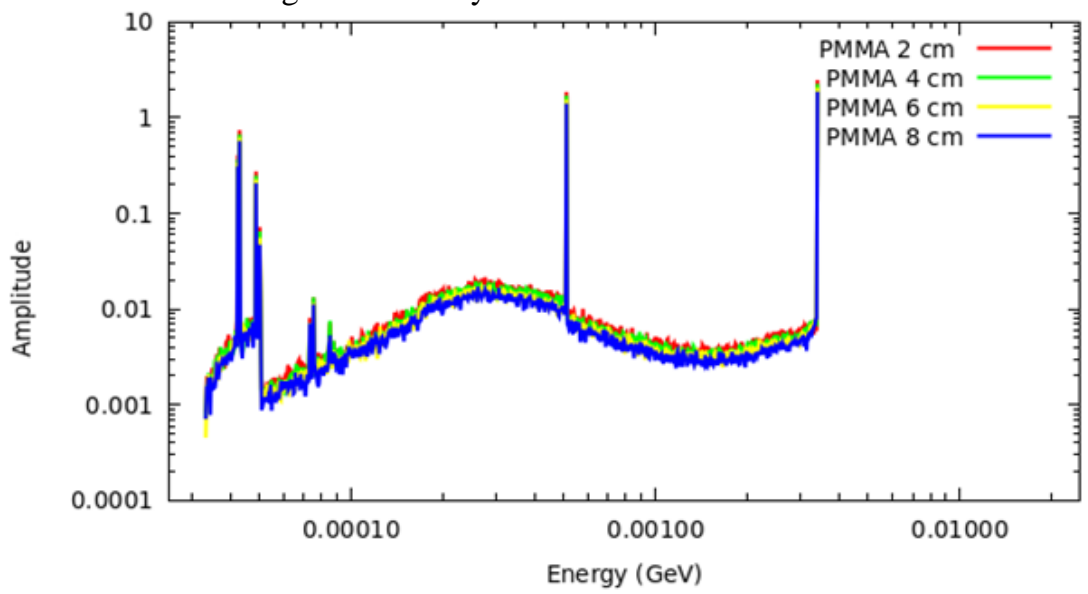

Figure 4: Transmitted photon profile across the simulation geometry showing phantom, anti-

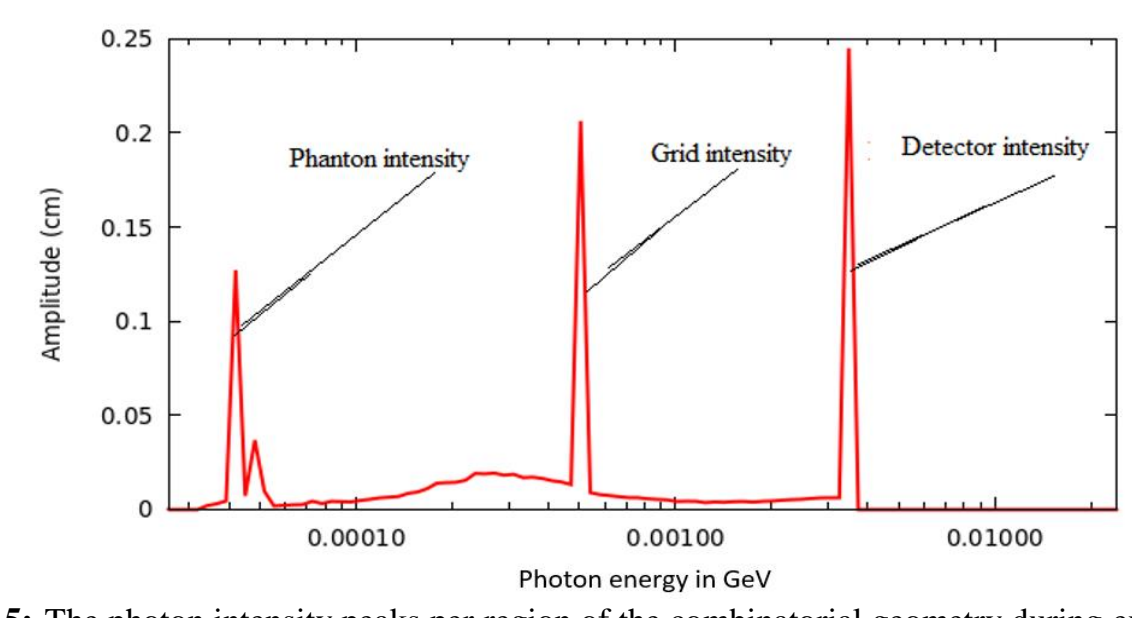

Figure 5: The photon intensity peaks per region of the combinatorial geometry during exposure of PMMA phantom. produce high quality images devoid of both system and quantum noises. The energy peaks were normalized to lower energy spectra $(1 / \mathrm{MeV})$ and to photon dose in Gy (1/Gy) as shown in Figure 6. Normalization allows for amplification of signals for easier spectra analysis. The normalization in Figure 6 (a) was to $1 / \mathrm{MeV}$ to correspond to the norminal diagnostic X-ray energies. The FLUKA code input files alow for photon energies inputs only in $\mathrm{GeV}$. The $1 / \mathrm{Gy}$ normalization was to convert the photon intensities to absorbed dose in grays (Gy) as shown in Figure 6 (b).

\section{scatter grid and detector energy deposit spectra for varrying phantom thicknesses.}



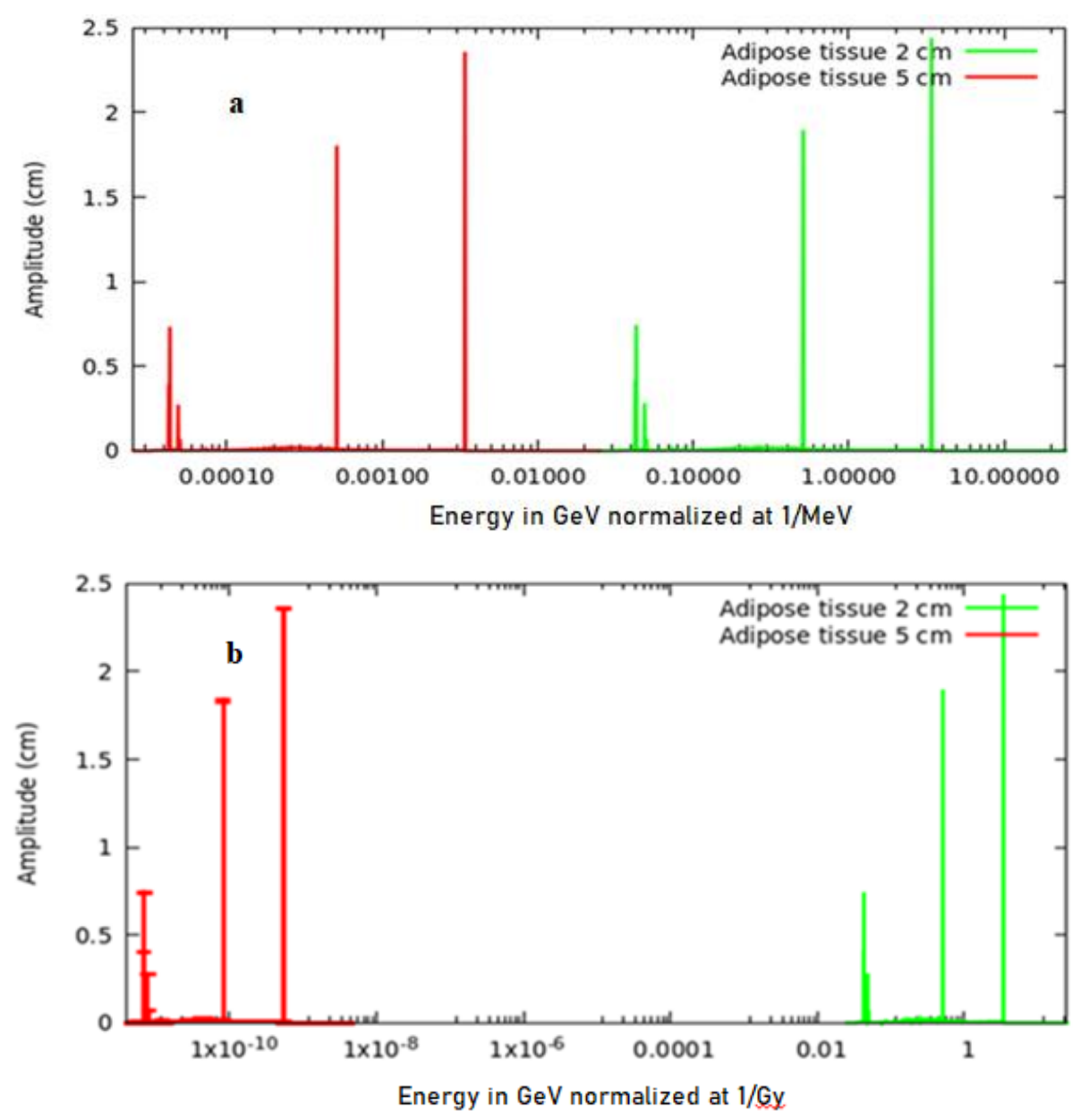

Figure 6: Varying spectra profiles for irradiation of adipose tissue of varying thickness normalized a) at $1 / \mathrm{MeV} \mathrm{X}$-ray energy, and b) at X-ray photon dose $1 / \mathrm{Gy}$.

\section{Discussion}

In this study, the scatter suppression algorithm's capabilities in scatter correction presented have been shown to be entirely dependent of the grid designs. Anti-scatter grid characteristics, mainly grid ratio, grid frequency, septa thickness and interspace, have been observed to significantly affect the grid performance efficacy in cutting off X-ray scatter. This observation is consistent with the findings of the studies by Fetterly and Schueler (2007) and Zhou et al. (2016). The flexibility in the FLUKA written user code enabled generation of grid designs of grid ratio between
5 and 21 having lead content values ranging between 0.1125 and $1.0522 \mathrm{~g} \mathrm{~cm}^{-2}$. Higher lead content of the septa significantly increases scatter cut-off and decreases photon septa penetration. This cumulatively reduces the scatter effects on the primary image signals. At constant exposure energy $(34 \mathrm{kV})$, scatter suppression increases with increasing grid ratio, lead to interspace ratio and grid septa thickness. Additionally, the use of high grid ratio anti-scatter grid enhances scatter correction efficiency. The results of the evaluation of grid performance by MC simulations through varying anti-scatter grids 
features has shown potency of significant reduction of scatter artefacts in projection radiography. Analysis of the simulated and experimental results generated Pearson's correlation coefficients of 0.983 and 0.981 for SF and SPR, respectively. This signifies strong correlations in the observed values validating the simulations protocols by the experimental results. The photon energy absorption and attenuation per region were observed to be dependent on the thickness of the phantom showing gradual increase in SPR and SF values as important grid performance indicators. However, the findings though limited to soft tissue equivalent phantom, have lent credence to the anti-scatter grids applications in digital radiography imaging systems for optimal scatter corrections using appropriate grid designs generated in this study. The FLUKA particle and transport code is therefore validated over the experimental results observed in this study for scatter suppression method in the X-ray imaging systems.

\section{Conclusion}

The simulation protocol described in this study has been validated by the experimental results obtained in this study and previous results by Omondi et al. (2020). The scatter suppression method was found to be effective in cutting-off significant X-ray scatter. However, the primary cut-off was minimal with an improvement in the anti-scatter grid designs for mammographic and general applications. Therefore, the validation of the simulation protocols engenders application in optimizing grid designs and investigating anti-scatter grids performance. The SPR and SF values obtained are comparable and in agreement with previous studies establishing the efficacy of the scatter suppression algorithm in scatter correction.

\section{Conflict of Interest}

The authors declare no conflict of interest.

\section{Acknowledgements}

The authors acknowledge the financial support granted for this study by the proprietor of
Breaking Limits Enterprises Mrs. Lilian Omondi, who sanctioned unlimited access to financial resources to meet the budget requirements. We acknowledge Muhimbili National Hospital for granting us access, ethical clearance and permission to use radiology department facilities. We also appreciate the radiographers of Mloganzila Hospital for their technical assistance and Innocent Junior for software programming. This work is forever dedicated to the Late Prof. Peter Kitau Msaki whose valuable guidance we still greatly miss. May his soul Rest in Eternal Peace.

\section{References}

Carton AK, Acciavatti R, Kuo J and Maidment ADA 2009 The effect of scatter and glare on image quality in contrast-enhanced breast imaging using an a-Si/CsI(Tl) fullfield flat panel detector. Med. Phys. 36(3): 920-928.

Chan H-P and Doi K 1982 Investigation of performance of anti-scatter grids: Monte Carlo simulation studies. Phys. Med. Biol. 27: 785-803.

Chan HP, Lam KL and Wu Y 1990 Studies of performance of antiscatter grids in digital radiography: Effect on signal to noise ratio. Med. Phys. 17: 655-664.

Del Lama LS, Cunha DM and Poletti ME 2017 Validation of a modified PENELOPE Monte Carlo code for applications in digital and dual-energy mammography. Rad. Phys. Chem. 137: 151-156.

Ferrari A, Sala PR, Fasso`A and Ranft J 2005 FLUKA: a multi-particle transport code CERN-2005-10, INFN/TC_05/11, SLACR-773.

Fetterly KA and Schueler BA 2008 Physical evaluation of prototype high-performance anti-scatter grids: potential for improved digital radiographic image quality. Phys. Med. Biol. 54: N37-42.

Fetterly KA and Schueler BA 2007 Experimental evaluation of fiber interspaced anti-scatter grids for large 
patient imaging with digital $\mathrm{x}$-ray systems. Phys. Med. Biol. 52: 4863-4880.

Omondi SO, Msaki PK, Kazema RR and Lugendo IJ 2020 Improved image quality in digital mammography using anti-scatter grids. Tanz. J. Sci. 46(3): 620-627.

Salvagnini E, Bosmans H, Struelens L and Marshall NW 2012 Quantification of scattered radiation in projection mammography: four practical methods compared. Med. Phys. 39: 3167-3180.

Sisniega A, Zbijewski W, Badal A, Kyprianou IS, Stayman JW, Vaquero JJ and Siewerdsen JH 2013 Monte Carlo study of the effects of system geometry and antiscatter grids on cone-beam CT scatter distributions. Med. Phys. 40(5): 051915.

Stankovic U, Ploeger LS, van Herk M and Sonkea JJ 2017 Optimal combination of anti-scatter grids and software correction for CBCT imaging. Med. Phys. 44(9): 4437-4451.

Vlachoudis V 2009 FLAIR: a powerful but user-friendly graphical interface for
FLUKA in Proceedings of the International Conference on Mathematics Computational Methods ( $M$ \& $C$ '09), Saratoga Springs, NY, USA.

Wang L, Shi L, Wang D 2012 Scatter Correction in Cone-beam CT Based on Deconvolution Proc. IEEE-EMBS Int. Conf. Biomed. Health Inf. (BHI 2012) Hong Kong and Shenzhen, China, 2-7 Jan 2012.

Zhou A, Yin Y, White GL and Davidson R 2016 A new solution for radiation transmission of anti-scatter grids. Biomed. Phys. Eng. Express 2: 055011.

Zhou A, White GL and Davidson R 2018 Validation of Monte Carlo code system for grid evaluation with interference effect on Rayleigh scattering. Phys. Med. Biol. 63(3): p. 03NT02.

Zhou A, Yin Y, Tan Q, L. White G, Davidson R 2020 The determination of the optimal strip-thickness of anti-scatter grids for a given grid ratio and strip height. Int. J. Imag. Syst. Technol. 30: 916-925. 